\title{
On Dominance and Context-dependence in Decisions Involving Multiple Attributes*
}

\author{
Prasanta K. Pattanaik \\ Department of Economics,University of California, \\ Riverside, CA 92521, U.S.A. \\ E-mail: prasanta.pattanaik@ucr.edu; Telephone: (951) 827 1592; \\ Fax: (951) 8275685 \\ Yongsheng $\mathrm{Xu}$, \\ Department of Economics, Andrew Young School of Policy Studies, \\ Georgia State University, Atlanta, GA 30303, U.S.A. \\ $\&$ \\ Central University of Finance and Economics, China. \\ E-mail: yxu3@gsu.edu; Telephone: (404) 413 0158; \\ Fax: 4044130145
}

This version: 19 November 2008

[Please do not cite this paper without the consent of at least one of the authors.]

*Earlier versions of this paper were presented in a conference at Oxford Poverty and Human Development Initiative, University of Oxford, and a conference at University of California, Riverside. We are grateful to the participants in these two conferences for many helpful comments. In particular, we would like to acknowledge our intellectual debt to Salvador Barberà, Kaushik Basu, Walter Bossert, Rajat Deb, Bhaskar Dutta, Indranil Dutta, Peter Hammond, Kotaro Suzumura, Mozaffar Qizilbash, and Thomas Schwartz. 


\section{Introduction}

In this paper, we discuss a difficulty in decision-making when there are multiple evaluative attributes in terms of which the options are assessed. We start with an informal outline of the difficulty and some examples from various sources in welfare economics, social choice theory, and individual decisionmaking in both ethical and non-ethical matters.

Imagine that you face the problem of choosing between two options, $x$ and $y$. How do you proceed in making your choice? A plausible route will be to identify first the evaluative attributes or criteria that you consider to be relevant for making your choice. Your choice between the two options will depend on these criteria identified by you. Typically, you would then commit yourself to the dominance principle with respect to the criteria that you have already identified, so that, whenever option $x$ is better than option $y$ in terms of some criteria and no worse than option $y$ in terms of any criterion, then, overall, you would consider option $x$ to be better than option $y$, and, hence, you would choose $x$ over $y$. When the dominance principle is applicable, there does not seem to be any need for worrying about the relative importance of the different criteria that you have identified. What happens if neither of the two options dominates the other in terms of the evaluative criteria? In particular, what happens if, in terms of some of the evaluative criteria,option $x$ is better than option $y$, and, in terms of some other evaluative criteria, option $y$ fares better than option $x$ ? In such cases, you would need to determine the relative importance or trade-offs between these different criteria. In determining how to make such tradeoffs, however, you may like to take into account the contextual features of $x$ and $y$. These contextual features do not constitute independent criteria for judging the options in any usual sense of the term 'criterion'; in fact, the notion of 'more' or 'less' (or, 'more desirable' or 'less desirable') with reference to these contextual features may not make any sense at all . Nevertheless, you may consider these contextual features to be be relevant for how you would like to trade off the gains in terms of some of the criteria against the losses in terms of some other criteria that you have identified at the outset. Your choice in the presence of conflicting criteria may then depend on these contextual features: if the contextual features are of one kind, then you would choose $x$ over $y$, and if they are of another kind, then you would choose $y$ over $x$. In what follows, such dependence on contextual features 
when neither option dominates the other in terms of the selected criteria will be called context-dependence ${ }^{1}$.

If this is how you make your decisions, going first by the dominance principle, defined with reference to the evaluative criteria, if at all the principle is applicable, but allowing contextual features to influence the relative importance that you attach to the different evaluative criteria when the principle of dominance is not applicable, then your preferences cannot be simultaneously transitive and continuous. This is the basic message that emerges from several examples of decision-making to be found in both economics and philosophy. How can this be? Consider the following informal reasoning. By context-dependence, there are options $x, y, z$ and $w$ such that

(i) $x$ and $z$ perform equally well in terms of the evaluative criteria, and so do $y$ and $w$, and yet, given that the selected criteria conflict when comparing $x$ and $y$ (and also when comparing $z$ and $w$ ), suppose you prefer $x$ to $y$ and $w$ to $z$ because either the contextual features of $x$ and $z$ are different or those of $y$ and $w$ are different and you allow these contextual features to determine how you trade off the gains and losses in terms of the different conflicting criteria. .

(ii) Assume that all the evaluative attributes refer to desirable attributes (for clarificatory comments on this assumption, see Section 3.1). We can then consider $z^{\prime}$, which has slightly more of some evaluative attribute than $z$ but exactly the same amount of each evaluative attribute as $z$, and $w^{\prime}$, which has slightly less of some evaluative attribute than $w$ and exactly the same amount of every other evaluative attribute as $w$. Since the difference between $z$ and $z^{\prime}$ and also the difference between $w$ and $w^{\prime}$ are assumed to be very small and you preferred $w$ to $z$ to start with, you will prefer $w^{\prime}$ to $z^{\prime}$ because your preferences over the options are continuous.

(iii) By dominance you must prefer $z^{\prime}$ to $x$ and $y$ to $w^{\prime}$.

(iv) Thus, you prefer $x$ to $y, y$ to $w^{\prime}, w^{\prime}$ to $z^{\prime}$, and $z^{\prime}$ to $x$, so that you have a cycle of strict preferences.

\footnotetext{
${ }^{1}$ For alternative formal formulations of this intuitive notion of context-dependence, see Section 3 below.
} 
The difficulty presented in the above reasoning is not isolated; it emerges in many decision-making problems in very different areas. We give several examples from the existing literature.

Example 1 (Comparison of living standards). Suppose we have some real indicators of living standards, or, functionings, to use the terminology of Sen $(1985,1987))$, such as health, education, nutrition, etc. Using these functionings, all of which represent desirable evaluative attributes or criteria, we want to compare the living standards of a given individual in different situations and also the living standards of different individuals. In this context, it has been suggested that, whenever an individual is doing better than another individual in terms of some of the functionings and is not doing worse in terms of any one of the functionings, the overall living standard of the first individual should be deemed to be at least as high as the living standard of the second individual, irrespective of the values and/ or the social and cultural backgrounds of the two individuals (this is the principle of dominance). If, however, $x$ and $y$ are two functioning bundles one of which has more of some functionings and the other has more of some other functionings, then, in comparing a given individual's standard of living from the two functioning bundles, $x$ and $y$, one can take a 'relativistic' position and allow the values of the individual under consideration and the norms and mores prevailing in her society to play some role in weighing the gains in terms of some functionings against the losses in terms of some other functionings (context-dependence); Pattanaik and $\mathrm{Xu}$ (2007) called such context-dependence in standard of living comparisons "minimal relativism". From our earlier reasoning, it then follows that the comparison of living standards cannot be transitive and continuous. At the risk of emphasizing the obvious, note that the culture or social background of a person is not a criterion in its own right. It does not admit the notion of 'more' or 'less'; nor does the appeal to culture in the decision process imply any judgment that one culture or social background is better or more desirable than another. It does, however, influence the trade-offs between the different evaluative attributes, i.e., the functionings, in our example.

The difficulty is not confined to comparisons of living standards based on real indicators. Consider the following

Example 2 (The Pareto principle and non-utility considerations). A basic value judgment, widely used in welfare economics, is the Pareto 
principle, which requires that, if a social state $x$ offers more utility to some individuals in the society as compared to another social state $y$ and no less utility to any individual as compared to $y$, then $x$ is socially better than $y$; this is the principle of dominance here, the individuals' utilities being the evaluative attributes. If, however, some individual, $i$, has higher utility in $x$ than in $y$ and some other individual, $j$, has higher utility in $y$ than in $x$, then the Pareto principle does not apply. In these cases where the Pareto principle does not apply, it seems tempting to allow non-utility considerations to influence the issue of how one should weigh one individual's loss of utility against another individual's gain in utility. Thus, if $x$ and $y$ differ only with respect to some aspect that relates to $i$ 's personal life, then one can take the position that the consideration of $i$ 's utilities from $x$ and $y$ must take strict priority over the consideration of other individisuals' utilities and $x$ should be considered socially better than $y$, and, on the other hand, if the two states differ only with respect some aspect relating to $j$ 's private life then, by a similar intuition, $y$ should be considered socially better than $x$ (see Sen (1970a, 1970b)). This, of course is an example of context-dependence here. It follows that such context-dependence based on the notion of the private life of an individual and the dominance rule embodied in the Pareto principle cannot be both satisfied if the social ranking is transitive and continuous in the individual utilities. ${ }^{2}$ Again, note that the physical descriptions of the social states, which determine whether two social states differ solely with respect to the private life of a given individual, do not constitute an independent criterion for judging the social states. Nevertheless, these physical descriptions may influence our judgment about whether we should give priority to one individual's gain (resp. loss) of utility over another individual's loss (resp. gain) in utility.

The difficulty goes beyond welfare economics and the theory of social choice. ${ }^{3}$ Consider the next example due to Hare (2007).

Example 3 (Individual decision-making in non-ethical matters). Consider an individual assessing the relative desirability of cars, using several criteria. The individual assesses cars in terms of two evaluative attributes, comfort and style. If one car is at least as good as the another car with re-

\footnotetext{
${ }^{2}$ For a discussion of a somewhat related result, see Kaplow and Shavell (2001).

${ }^{3}$ See Fleurbaey (2007) and Weymark (2008) for some related examples in welfare economics and the theory of social choice.
} 
spect to both comfort and style and is strictly better than the other car with respect to at least one of these two criteria, then he ranks the first car higher than the second in an overall comparison of the two cars (dominance). If, however, there is a conflict between the rankings of the two cars in terms of comfort and style, then he attaches a greater weight to comfort if the two cars happen to be two different Mercedes (as Hare puts the individual's reasoning here, "comfort is what a Mercedes is really about") but a lower weight to comfort if the two cars happen to be Bentley (as Hare captures the individual's feelings, "style is what a Bentley is really about"). Though the brand of the car is not an independent evaluative criterion in this example, it does influences the comparisons of cars in some cases by influencing the relative importance that the agent attaches to the two evaluative attributes, namely, comfort and style.This, of course, is an instance of context-dependence. In such a case, the individual cannot have transitive and continuous preferences over the cars.

And finally, consider the following example which is a slightly modified version of an example due to Hare (2007).

Example 4 (Individual decision-making in ethical matters). Here the agent faces a moral problem. There are several persons who are in distress and need help. The agent is faced with the problem of ranking, in terms of his ethical obligations, the options of helping these different individuals and also the option of helping nobody. There are three evaluative attributes: the urgency of a person's need for help (the more urgent the need, the greater the moral obligation of the agent to help) ; the extent to which the person in distress is responsible for his own situation (the less the person's responsibility, the grater the obligation of the agent to help); and the cost involved in helping the person in distress (the lower the cost, the greater the agent's moral obligation). In ranking the options of helping either of two individuals, the agent goes first by the dominance principle defined in terms of these three criteria if the dominance principle is applicable at all. If, however, these criteria conflict in some comparisons, then the agent needs to assess the relative importance of these different criteria. In doing so, he looks at contextual features such as whether the person in need is an adult member of his family or the person in need of help is a very young and inexperienced person who happens to be a stranger or the person is a very young and inexperienced family member, and so on. If the person happens 
to be young and inexperienced, the criterion of responsibility is given relatively less importance; on the other hand, if the person is a relation rather than a stranger, the criterion of cost is given relatively less importance. In considering these contextual features when the three moral criteria conflict, the agent does not not use the contextual features as independent moral criteria; instead, he allows them to bear on the relative importance that he attaches to his evaluative attributes, the urgency of need of the person, the extent to which the person is responsible for his plight, and the cost involved in providing help. In this case, again, the individual cannot have a transitive and continuous ranking over the options. Note that one of the evaluative attributes here, namely, the cost to the decision-maker, is, intuitively, an undesirable attribute, but, as we explain in Section 3.1, we can formally replace it by a suitably defined desirable evaluative attribute without losing any part of the underlying intuition.

In all these examples, we face a tension between the principle of dominance, the use of context-dependence in resolving conflicts among the criteria, and the requirement that the overall ranking of options should be transitive and continuous. While such tension manifests itself in different ways in these different areas, there is a common formal structure involved in all these instances. The main purpose of this paper is to propose a general analytical framework in which this formal structure can be clarified. In the process, we provide general results which imply, as special cases, the results of Pattanaik and Xu (2007) and Hare (2007) as well as a version of Sen's (1970a, 1970b) paradox of the Paretian liberal. We also prove a related result that extends the analysis of Kaplow and Shavell (2001). The remainder of the paper is organized as follows. In Section 2, we develop a framework for our analysis. Section 3 introduces several properties of the ranking of the options. We present our main results in Section 4. In Section 5, we give some examples to illustrate the roles played by the different conditions postulated in one of our main results. Section 6 deals with some extensions of our results. A brief conclusion is given in Section 6 .

\section{The framework}

Let $C=\{1,2, \ldots, m\}$ be the set of all evaluative attributes or criteria $(\infty>$ $m \geqslant 2)$. For every evaluative attribute $j \in C$, let let $X_{j}$ be the set of all 
values that $j$ can take $\left(\# X_{j} \geqslant 2\right)$. The interpretation of these evaluative attributes will be different in different contexts as seen the examples given in Section 1.

Assumption 1. For every $j$ in $C$, there exists a linear ordering ${ }^{4} R_{j}$ defined over $X_{j}$. Further, for some attribute $j$ in $C, X_{j}$ is an interval $\left[\beta_{j}, \alpha_{j}\right]$, such that $-\infty \leqslant \beta_{j}<\alpha_{j} \leq \infty$ and, for all $s, t \in\left[\beta_{j}, \alpha_{j}\right], s R_{j} t$ iff $s \geqslant t$.

For every evaluative attribute $j$, it makes sense to speak of more or less of the attribute $j$, and the linear ordering $R_{j}$ formally captures this notion of more or less. The linear ordering $R_{j}$ stands for the binary relation of "offers at least as much of evaluative attribute $j$ as". For every $j$ in $C$, and for all $s, t$ in $X_{j}, s R_{j} t$ denotes that $s$ offers at least as much of attribute $j$ as $t$.

Let $D$ denote the set of those evaluative attributes $j$ for which $X_{j}$ is an interval $\left[\beta_{j}, \alpha_{j}\right]$, such that $-\infty \leq \beta_{j}<\alpha_{j} \leq \infty$ and for all $s, t \in\left[\beta_{j}, \alpha_{j}\right], s R_{j} t$ iff $s \geqslant t$. It may be noted that, given our Assumption 1, the set $D$ is nonempty. Under Assumption 1, it is possible to have evaluative attributes, which are ordinally measurable but which are not cardinally measurable. Thus, we may be able to distinguish between a higher level of health and a lower level of health, but we may not be able to measure health on a cardinal scale along a real interval. ${ }^{5}$

Let $X_{m+1}$ be the non-empty set of all alternative specifications of the contextual features in the problem under consideration. We assume that $\left|X_{j}\right| \geqslant 2$. Thus, in our Example 1 in Section 1, the culture and social background of the individual whose standard of living is under consideration constitute the contextual feature; similarly, in Example 2 there, the physical description of the social state constitutes the contextual feature.

Let $U$ denote $\{1,2, \ldots, m+1\}$. Let $X \equiv X_{1} \times X_{2} \times \cdots \times X_{m+1}$ be the set of all conceivable options or alternatives. Let $\succeq$ be a reflexive binary relation ("at least as good as") defined over $X$. The asymmetric and symmetric parts of $\succeq$ are denoted by $\succ$ and $\sim$, respectively. Thus, for all $x, y \in X, x \succeq y$ is to be interpreted as "alternative $x$ is at least as good as alternative $y$ ", $x \succ y$

\footnotetext{
${ }^{4}$ Recall that $R_{j}$ is a linear ordering over $X_{j}$ iff $R_{j}$ is reflexive, transitive, complete and anti-symmetric (i.e., for all $s, t \in X_{j},\left[s R_{j} t\right.$ and $\left.t R_{j} s\right] \Rightarrow s=t$ ).

${ }^{5}$ It is possible to relax somewhat the assumption that all attributes in $C$ are at least ordinally measurable. Thus, our formal results will not be affected if we replace Assumption 1 by the weaker assumption that, for some $j \in C, X_{j}$ is a real interval $\left[\beta_{j}, \alpha_{j}\right]$, such that $-\infty \leqslant \beta_{j}<\alpha_{j} \leq \infty$ (for all $s, t \in\left[\beta_{j}, \alpha_{j}\right], s$ denotes at least as much of attribute $j$ as $t$ iff $s \geqslant t)$.
} 
is to be interpreted as " $x$ is better than $y$ ", and $x \sim y$ is to be interpreted as " $x$ is indifferent to $y$ ". We say that $\succ$ is acyclic if and only if there does not exist a $\succ$-cycle in $X$, i.e., there do not exist $x^{1}, x^{2}, \ldots, x^{h} \in X$, such that $\left[x^{1} \succ x^{2}\right.$ and $x^{2} \succ x^{3}$ and $\ldots$ and $x^{h-1} \succ x^{h}$ and $\left.x^{h} \succ x^{1}\right]$. It is well-known that acyclicity of $\succ$ is a much weaker requirement than transitivity of $\succeq$.

Several specific frameworks developed in the literature may be noted as special cases of our general framework. In Pattanaik and $\mathrm{Xu}$ (2007), $C$ is assumed to be the set of all functionings and all functionings are assumed to be measurable on a ratio scale along a non-degenerate real interval, so that $D=C$. Pattanaik and $\mathrm{Xu}$ (2007) assume that the contextual features simply

refer to the culture and social background of the person whose standard of living is under consideration. In Example 2 in Section 1, the utilities are the evaluative attributes in $C$ and the physical descriptions of the social states are the contextual features. Analogous interpretations hold for Examples 3 and 4 in Section 1.

\section{Weak dominance and dominance, context- dependence, and weak continuity}

\subsection{Weak Dominance and Dominance}

We now introduce several alternative formulations of the dominance principle.

$\succeq$ satisfies weak dominance (resp.dominance) iff there exists $j \in D$ such that, for all $x$ and $y$ in $X$, if $x_{j}>y_{j}$ and $x_{i}=y_{i}$ for all $i \in C-\{j\}$, then $x \succeq y($ resp. $x \succ y)$.

Weak dominance and dominance implicitly assume that there is at least one 'desirable' evaluative attribute that is measured along a real interval. This is true for most of the examples that we discussed in Section 1. What if all the evaluative attributes in $D$ are, like the decision-maker's cost in Example 4 in Section 1, undesirable? This, however, is not a serious problem. Consider the evaluative attribute of cost to the decision-maker. Suppose, this cost can be anything between 0 dollars and 5000 dollars. Then, while modeling the problem, we can replace the evaluative attribute of cost by a formally specified evaluative attribute $g$ (if one likes, one can call it the 
decision-maker's monetary benefit), which can take any value in the interval $[-5000,0]$. An increase in $g$ will then be intuitively equivalent to a decrease in the cost and will make the option more desirable. In general, without any loss of intuition, an undesirable evaluative attribute, which is measured along a real interval $[\beta, \alpha]$, can be replaced in the formal model by a desirable evaluative attribute, which is measured along the real interval $[-\alpha,-\beta]$. A more complex modeling issue, however, arises when, intuitively, none of the evaluative attributes, which are measured along real intervals, is either desirable over the entire range of its permissible values or undesirable over the entire range of its permissible values (so that, starting from some initial quantity of the attribute, an increase in the amount of the attribute is desirable while starting from some other initial quantity, an increase in the amount of the attribute is undesirable). We consider this case in Section 6.

\subsection{Context-dependence}

The basic intuitive idea of context-dependence is that, though contextual features do not constitute evaluative criteria on their own, they may, nevertheless, influence the ranking of options by affecting the relative importance to be attached to the different evaluative criteria in the absence of dominance in terms of these criteria. One can, however, think of several alternative formulations of this intuitive idea. We consider three alternative versions in the following definitions.

$\succeq$ satisfies context-dependence (type I) iff there exist $a, b, c, d$ in $X$, such that [for all $j$ in $C, a_{j}=c_{j}$ and $\left.b_{j}=d_{j}\right],\left[a_{m+1}=b_{m+1}\right.$ and $\left.c_{m+1}=d_{m+1}\right]$, and $[a \succ b$ and $d \succ c]$.

$\succeq$ satisfies context-dependence (type II) iff there exist $a, b, c, d$ in $X$, such that [for all $j$ in $C, a_{j}=c_{j}$ and $\left.b_{j}=d_{j}\right]$, and $[a \succ b$ and $d \succ c$ ].

$\succeq$ satisfies context-dependence (type III) iff there exist $x, y \in X$, such that $\left(x_{j}=y_{j}\right.$ for all $\left.j \in C\right)$ and $x \succ y$.

Context-dependence(type I) stipulates the existence of four alternatives $a, b, c$, and $d$, such that $a$ and $c$ are indistinguishable in terms of the evaluative properties and so are $b$ and $d, a$ and $b$ have the same contextual features and so do $c$ and $d$, and, overall, $a$ ranks higher than $b$ and $d$ ranks higher than $c$. Presumably, this happens because the contextual features common to $a$ and 
$b$ affect the relative importance of the evaluative criteria in a different way as compared to the contextual features shared by $c$ and $d$. Context-dependence (type II) is somewhat weaker than context-dependence (type I), insofar as context-dependence (type II) does not stipulate that the contextual features must be the same for $a$ and $b$, as well as for $c$ and $d$. Context-dependence (type III) is not logically comparable with context-dependence (type I). It is, however, stronger than context-dependence (Type II). To see this, consider $x, y \in X$, such that $\left(x_{j}=y_{j}\right.$ for all $\left.j \in C\right)$ and $x \succ y$. Then, letting $a=d=x$ and $b=c=y$, it it is clear that $a, b, c$, and $d$ satisfy the conditions specified in the definition of context-dependence (type II) and we have $a \succ b$ and $d \succ c$.

\subsection{Weak continuity in attributes in $D$}

We now introduce two weak properties of continuity of $\succeq$ in attributes in $D$ (recall that every attribute in $D$ is measured along a real interval).

$\succeq$ satisfies weak continuity in attributes in $D$ iff, for all $x$ and $y$ in $X$, and, for all all $j \in D,\left(\left[x \succ y\right.\right.$ and $x_{j}>\beta_{j}$ ] implies [ for some $x^{\prime}$ in $X, x_{j}^{\prime}<x_{j}$, $x_{k}=x_{k}^{\prime}$ for all $k \in U-\{j\}$, and $\left.\left.x^{\prime} \succ y\right]\right)$ and $\left(\left[x \succ y\right.\right.$ and $\left.y_{j}<\alpha_{j}\right]$ implies [for some $y^{\prime}$ in $X, y_{j}^{\prime}>y_{j}, y_{k}^{\prime}=y_{k}$ for all $k \in U-\{j\}$, and $\left.x \succ y^{\prime}\right]$ ).

$\succeq$ satisfies restricted weak continuity in attributes in $D$ iff, for all $x$ and $y$ in $X$ such that $x_{m+1}=y_{m+1}$, and, for all all $j \in D,\left(\left[x \succ y\right.\right.$ and $\left.x_{j}>\beta_{j}\right]$ implies [ for some $x^{\prime}$ in $X, x_{j}^{\prime}<x_{j}, x_{k}=x_{k}^{\prime}$ for all $k \in U-\{j\}$, and $\left.x^{\prime} \succ y\right]$ ) and $\left(\left[x \succ y\right.\right.$ and $\left.y_{j}<\alpha_{j}\right]$ implies [for some $y^{\prime}$ in $X, y_{j}^{\prime}>y_{j}, y_{k}^{\prime}=y_{k}$ for all $k \in U-\{j\}$, and $\left.\left.x \succ y^{\prime}\right]\right)$.

Weak continuity of $\succeq$ in attributes in $D$ is weaker than continuity, defined in the usual fashion ${ }^{6}$, of $\succeq$ in the attributes in $D$. Restricted weak continuity is even weaker, insofar as its scope is restricted only to the ranking (in terms of $\succeq$ ) of options all of which have the same contextual features.

\section{Some Impossibility results}

We now investigate implications of combining in different ways the properties of $\succeq$ introduced earlier. The following propositions constitute our main

\footnotetext{
${ }^{6}$ See Section 4.
} 
findings.

Proposition 1. No transitive $\succeq$ can simultaneously satisfy weak dominance, context-dependence (type I), and restricted weak continuity in attributes in D.

The formal proof of Proposition 1 is given in Appendix A. The basic structure of the proof can, however, be outlined here, using a special case. Let $C=D$ and let $C$ contain exactly two evaluative attributes and let both these attributes be 'desirable' in the sense explained earlier in Section 3.1 (in the formal proof, these restrictive assumptions are not needed). Given context-dependence (type I), let $a, b, c$, and $d$ be such that $\left[a_{1}=c_{1}, a_{2}=\right.$ $c_{2}, b_{1}=d_{1}, b_{2}=d_{2}, a_{3}=b_{3}$, and $\left.c_{3}=d_{3}\right]$, and $(a \succ b$ and $d \succ c)$. For the sake of simplicity, assume that for all $j \in\{1,2\}$, each of $a_{j}, b_{j}, c_{j}$, and $d_{j}$ is greater than $\beta_{j}$ (note that this assumption is not required for the formal proof which we give in Appendix $A$ ). Then, by restricted weak continuity of $\succeq$ in attributes in $D$, for some $a^{\prime}$, which has less of evaluative attributes 1 and 2 than $a$ and $c$, and some $d^{\prime}$, which has less of attributes 1 and 2 than $b$ and $d$, we must have $a^{\prime} \succ b$ and $d^{\prime} \succ c$. But then, by weak dominance, $c \succeq a^{\prime}$ and $b \succeq d^{\prime}$. Together, we have $\left(a^{\prime} \succ b, b \succeq d^{\prime}, d^{\prime} \succ c\right.$ and $\left.c \succeq a^{\prime}\right)$, which contradicts the transitivity of $\succeq$.

Proposition 1 is a generalization of Proposition 1 of Pattanaik and $\mathrm{Xu}$ (2007) (see Example 1 in Section 1 above).

Proposition 1 requires $\succeq$ to be transitive. As the next proposition shows, transitivity of $\succeq$ can be weakened to acyclicity of $\succ$ if weak dominance is strengthened to dominance; while dominance is stronger than weak dominance, intuitively, the former property is hardly less plausible than the latter.

Proposition 2. No $\succeq$ such that $\succ$ is acyclic can simultaneously satisfy dominance, context-dependence (type I), and restricted weak continuity in attributes in $D$.

The proof of Proposition 2 is similar to that of Proposition 1, and we omit it. We note that, in the above illustration of Proposition 1, if dominance is used for $b$ and $d^{\prime}$ and for $c$ and $a^{\prime}$, then we will get $b \succ d^{\prime}$ and $c \succ a^{\prime}$. Together with $a^{\prime} \succ b$ and $d^{\prime} \succ c$, a cycle is obtained, which contradicts the acyclicity of $\succ$.

Hare's (2007) analysis (see Examples 3 and 4 in Section 1 above) constitutes a special case of Proposition 2. 
Proposition 3 below differs from Proposition 1 in so far as Proposition 3 replaces context-dependence (type I) and restricted weak continuity by context-dependence (type II) and weak continuity, respectively. Propositions 2 and 4 differ in an analogous fashion.

Proposition 3. No transitive $\succeq$ can simultaneously satisfy weak dominance, context-dependence (type II), and weak continuity in attributes in $D$.

Proposition 4. No $\succeq$ such that $\succ$ is acyclic can simultaneously satisfy dominance, context-dependence (type II), and weak continuity in attributes in $D$.

The proofs of Propositions 3 and 4 are very similar to the proofs of Propositions 1and 2, respectively; therefore, we omit the proofs of Propositions 3 and 4.

Proposition 4 implies a variant of Sen's (1970a, 1970b) famous impossibility of the Paretian liberal, where the dominance rule takes the form of the Pareto criterion and context-dependence (type II) takes the form of Sen's condition of minimal liberalism. It may, however, be noted that, in this variant of Sen's impossibility of the Paretian liberal, Sen's original condition that the the set of all possible profiles of individual orderings over the options be the domain of the social decision rule is replaced by weak continuity of the social ranking of all options.

Our final result in this section shows the incompatibility of weak dominance and context-dependence (type III) in the presence of weak continuity.

Proposition 5. $\succeq$ cannot simultaneously satisfy weak dominance, contextdependence (type III), and weak continuity in attributes in $D$.

The proof of Proposition 5 is given in Appendix A. Proposition 5 constitutes a generalization of the result of Kaplow and Shavell (2001).

\section{Some Examples}

Some examples may be helpful in clarifying the intuitive structure of the propositions in the preceding section and also in demonstrating the independence of the restrictions on $\succeq$ postulated in these propositions. For the 
sake of brevity, we consider only Proposition 1, which shows the impossibility of finding a transitive $\succeq$ that simultaneously satisfies weak dominance, context-dependence (type I), and restricted weak continuity in attributes in $D$.

Assume that $C=D=\{1,2\}$. Let $X_{1}=X_{2}=[0,100]$ and let $X_{3}=\{p, r\}$. We consider four examples.

(i) Let $\succeq_{1}$ be a binary relation over $X$, such that, for all $x, y \in X, x \succeq_{1} y$ iff $3 x_{1}+7 x_{2} \geqslant 3 y_{1}+7 y_{2}$. It can be checked that $\succeq_{1}$ satisfies reflexivity, transitivity, weak dominance, and restricted weak continuity but violates context-dependence (type I).

(ii) Let $u$ be a real valued function defined over $X$ such that, for all $x \in X$, [if $x_{3}=p$, then $u(x)=3 x_{1}+7 x_{2}$ ] and [if $x_{3}=r$, then $u(x)=9 x_{1}+x_{2}$ ]. Let $\succeq_{2}$ be a binary relation over $X$, such that, for all $x, y \in X, \quad x \succeq_{2} y$ iff $u(x) \geqslant u(y)$. It can be easily seen that $\succeq_{2}$ satisfies reflexivity, transitivity, context-dependence (type I), and restricted weak continuity. But $\succeq_{2}$ violates weak dominance. To see the violation of weak dominance, consider $a, b \in X$, such that $a_{1}=10, a_{2}=4, a_{3}=p, b_{1}=9, b_{2}=3$, and $b_{3}=r$. Then $a$ dominates $b$ in terms of the evaluative criteria, but $b \succ_{2} a$.

(iii) Let $\succeq_{3}$ be a binary relation defined over $X$, such that for all $x, y \in X$, $x \succeq_{3} y$ iff $\left[x_{1}+x_{2}>y_{1}+y_{2}\right]$ or $\left[x_{1}+x_{2}=y_{1}+y_{2}\right.$ and $u(x) \geqslant y(y)$, where $u$ is the real-valued function over $X$ specified in (ii) above]. It can be checked that $\succeq_{3}$ satisfies reflexivity, transitivity, weak dominance, and context-dependence (type I) but violates restricted weak continuity.

(iv) Let $\succeq_{4}$ be a binary relation defined over $X$ such that, for all $x, y \in X$, [if $x_{3}=y_{3}=p$, then $x \succeq_{4} y$ iff $3 x_{1}+7 x_{2} \geqslant 3 y_{1}+7 y_{2}$ ], [if $x_{3}=y_{3}=r$, then $x \succeq_{4} y$ iff $7 x_{1}+3 x_{2} \geqslant 7 y_{1}+3 y_{2}$ ], and [if $x_{3}=p$ and $y_{3}=r$, then $\left(x \succeq_{4} y\right.$ iff $\left.\left.5 x_{1}+5 x_{2} \geqslant 5 y_{1}+5 y_{2}\right)\right]$. It can be checked that $\succeq_{4}$ satisfies reflexivity, weak dominance, context-dependence (type I), and restricted weak continuity. $\succeq_{4}$, however, violates transitivity. To see the violation of transitivity, consider $a, b, c \in X$, such that $a_{1}=10, a_{2}=5, a_{3}=p, b_{1}=5, b_{2}=10, b_{3}=r, c_{1}=$ $14, c_{2}=1$, and $c_{3}=p$. It can be checked that $a \sim b$ and $b \sim c$ and $a \succ c$.

Examples (i) through (iv) above show that transitivity, weak dominance, context-dependence (type I), and restricted weak continuity are independent properties of $\succeq$. 


\section{Further on weak dominance and domi- nance}

Though weak dominance and dominance are plausible properties, they have one restrictive feature. They are based on the implicit assumption that, in our intuitive description of the problem, either there exists an evaluative attribute, which is measured along a real interval and which is desirable over the entire range of its values, or there exists an evaluative attribute, which is measured along a real interval and which is undesirable over the entire range of its values so that it can be replaced by a suitably defined evaluative attribute which is desirable over the entire range of its values. While this is true of all the examples that we discussed in Section 1 and we do not know of any example in the existing literature that violates this intuitive assumption, one can nevertheless think of examples where this may not be true. Suppose we have an analytical framework where the well-being of a person depends on the level of her calorie consumption, among other things, and calorie consumption is the only attribute in $D$. It is possible that, intuitively, an increase in the level of calorie consumption is considered desirable until it reaches the level of 2500 calories, but, any further increase beyond that is undesirable ${ }^{7}$. In this case, weak dominance and dominance, as we have defined these properties earlier, would not be plausible ${ }^{8}$. It is, however, possible to weaken the properties of weak dominance and dominance to permit such cases. Consider the following weaker versions of the two properties.

$\succeq$ satisfies local weak monotonicity iff, there exists $j \in D$, such that, for all $x$ in $X$, there exists $\epsilon>0$ such that

$x_{j}<\alpha_{j}$ implies either $\left[y \succeq x\right.$ for all $y \in X$ with $x_{j}<y_{j}<x_{j}+\epsilon$ and $y_{k}=x_{k}$ for all $k \in C-\{j\}]$, or $\left[x \succeq y\right.$ for all $y \in X$ with $x_{j}<y_{j}<x_{j}+\epsilon$ and $y_{k}=x_{k}$ for all $\left.k \in C-\{j\}\right]$,

and

\footnotetext{
${ }^{7}$ We are grateful to Sudhir Anand for this example.

${ }^{8}$ Note that no such intuitive problems regarding weak dominance or dominance in core properties arises in Sen's functioning approach to living standards (see Example 1 in Section 1) since functionings are defined in such a way that they are all desirable attributes. Thus, calories would not be a functioning in Sen's sense though being well nourished would be so.
} 
$x_{j}>\beta_{j}$ implies $\left[y \succeq x\right.$ for all $y \in X$ with $x_{j}-\epsilon<y_{j}<x_{j}$ and $y_{k}=x_{k}$ for all $k \in C-\{j\}]$, or $\left[x \succeq y\right.$ for all $y \in X$ with $x_{j}-\epsilon<y_{j}<x_{j}$ and $y_{k}=x_{k}$ for all $\left.k \in C-\{j\}\right]$.

$\succeq$ satisfies local monotonicity iff, there exists $j \in D$, such that, for all $x$ in $X$, there exists $\epsilon>0$ such that

$x_{j}<\alpha_{j}$ implies either $\left[y \succ x\right.$ for all $y \in X$ with $x_{j}<y_{j}<x_{j}+\epsilon$ and $y_{k}=x_{k}$ for all $k \in C-\{j\}]$, or $\left[x \succ y\right.$ for all $y \in X$ with $x_{j}<y_{j}<x_{j}+\epsilon$ and $y_{k}=x_{k}$ for all $\left.k \in C-\{j\}\right]$,

and

$x_{j}>\beta_{j}$ implies $\left[y \succ x\right.$ for all $y \in X$ with $x_{j}-\epsilon<y_{j}<x_{j}$ and $y_{k}=x_{k}$ for all $k \in C-\{j\}]$, or $\left[x \succ y\right.$ for all $y \in X$ with $x_{j}-\epsilon<y_{j}<x_{j}$ and $y_{k}=x_{k}$ for all $\left.k \in C-\{j\}\right]$.

Clearly, local weak-monotonicity and local monotonicity do not involve the implicit assumption that every evaluative attribute, which is measured along a real interval, is either desirable over the entire range of its values or undesirable over the entire range of its values. They simply require the existence of an evaluative attribute $j$, such that $j$ is measured along a real interval, and, starting with any $x$ in $X$, either all small increases in the amount of $j$ make the option more attractive or all small increases in the amount of $j$ make the option less attractive; and similarly for small decreases in the amount of $j$.

Given these properties, we remark that the counterparts of Propositions $1,2,3,4$, and 5 using local weak-monotonicity and local monotonicity instead of weak dominance and dominance, continue to hold if we use the following continuity properties of $\succeq$ in attributes in $D$.

$\succeq$ satisfies continuity in attributes in $D$ iff, for all $j \in D$ and for all $x, y \in X$, if $x \succ y$ [resp. $y \succ x$ ], then there exists $\varepsilon>0$ such that, for all $x^{\prime} \in X$, if $\left|x_{j}-x_{j}^{\prime}\right|<\varepsilon$ and for all $i \in U-\{j\}, x_{i}^{\prime}=x_{i}$, then $x^{\prime} \succ y$ [resp. $y \succ x^{\prime}$ ].

$\succeq$ satisfies restricted continuity in attributes in $D$ iff, for all $j \in D$ and for all $x, y \in X$, if $x \succ y$ [resp. $y \succ x$ ] and $x_{m+1}=y_{m+1}$, then there exists $\varepsilon>0$ such that, for all $x^{\prime} \in X$, if $\left|x_{j}-x_{j}^{\prime}\right|<\varepsilon$ and for all $i \in U-\{j\}, x_{i}^{\prime}=x_{i}$, then $x^{\prime} \succ y$ [resp. $y \succ x^{\prime}$ ]. 
With these properties in hand, we can prove the following propositions; since their proofs are, in many ways, similar to the proofs of Propositions 1, $2,3,4$, and 5 , we omit them.

Proposition $\mathbf{1}^{\prime}$. No transitive $\succeq$ can simultaneously satisfy local weak monotonicity, context-dependence (type I), and restricted continuity in attributes in $D$.

Proposition $\mathbf{2}^{\prime}$. No $\succeq$ such that $\succ$ is acyclic can simultaneously satisfy local monotonicity, context-dependence (type I), and restricted continuity in attributes in $D$.

Proposition $3^{\prime}$. No transitive $\succeq$ can simultaneously satisfy local weak monotonicity, context-dependence (type II), and continuity in attributes in D.

Proposition $4^{\prime}$. No $\succeq$ such that $\succ$ is acyclic can simultaneously satisfy local monotonicity, context-dependence (type II), and continuity in attributes in $D$.

Proposition $\mathbf{5}^{\prime}$. No $\succeq$ can satisfy weak local monotonicity, contextdependence (type III), and continuity in attributes in $D$.

\section{Concluding remarks}

In decisions involving multiple criteria, there is a fundamental incompatibility between various dominance principles and the principles of contextdependence in the presence of rationality postulates and certain requirements of continuity. Dominance principles seem to be highly plausible in such situations. Principles of context-dependence are quite weak: a rejection of them would imply that contextual features should never have any influence on the relative importance to be attached to the different criteria when the dominance principle is not applicable. Rationality and continuity seem to be widely accepted requirements in decision theory. If we are not prepared to give up any of these properties in making choices, then we are faced with a dilemma. It would be interesting to examine and investigate further when and how some of these properties may be weakened to arrive at consistent decisions. 


\section{References}

Hare, C. (2007), "Rationality and the distant needy", Philosophy and Public Affairs, 35, pp. 161-178.

Fleurbaey, M. (2007), "Social choice and the indexing dilemma", Social Choice and Welfare, 29, pp. 633-648.

Kaplow, L. and S. Shavell, 2001, "Any non-welfarist method of policy assessment violates the Pareto principle", Journal of Political Economy, 109, 281-286.

Pattanaik, P.K. and Y. Xu, 2007, "Minimal relativism, dominance, and standard of living comparisons based on functionings", Oxford Economic Papers, 59, 354-374.

Sen, A. (1985). Commodities and Capabilities. Amsterdam: North-Holland.

Sen, A. (1987). The Standard of Living. Cambridge: Cambridge University Press.

Weymark, J. (2008), "Must one be an ogre to rationally prefer aiding the nearby to the distant needy?" Presidential Address, 9th International Meeting of the Society of Social Choice and Welfare, June 19-22, 2008, Concordia University, Montreal, Canada. 


\section{Appendix A}

Proof of Proposition 1. Suppose a transitive $\succeq$ satisfies weak dominance, context-dependence (type I), and restricted weak continuity in attributes in $D$. We shall show that this supposition leads to a contradiction.

By weak dominance, there exists an attribute $g \in D$, such that,

for all $x, y \in X$, if $x_{j}=y_{j}$ for all $j \in C-\{g\}$ and $x_{g}>y_{g}$, then $x \succeq y$.

Throughout the rest of the proof, we shall treat such $g$ as fixed.

By context-dependence (type I),

there exist $a, b, c, d \in X$, such that (for all $j \in C, a_{j}=c_{j}$ and $b_{j}=d_{j}$ ), $\left(a_{m+1}=b_{m+1}\right.$ and $\left.c_{m+1}=d_{m+1}\right)$, and $(a \succ b$ and $d \succ c)$.

We consider the following cases:

$a_{g}=c_{g}=\beta_{g}, b_{g}=d_{g}=\beta_{g} ;$

$a_{g}=c_{g}=\beta_{g}, b_{g}=d_{g}>\beta_{g} ;$

$a_{g}=c_{g}>\beta_{g}, b_{g}=d_{g}=\beta_{g}$;

and

$a_{g}=c_{g}>\beta_{g}, b_{g}=d_{g}>\beta_{g}$.

Suppose (3) holds. Then, by restricted weak continuity and $(a \succ b)$, there exists $b^{\prime} \in X$, such that, $b_{g}^{\prime}>b_{g}=d_{g}$ and $\left(b_{j}^{\prime}=b_{j}\right.$ for all $\left.j \in U-\{g\}\right)$ and $a \succ b^{\prime}$; and, by restricted weak continuity and $(d \succ c)$, there exists $c^{\prime} \in X$, such that, $c_{g}^{\prime}>c_{g}=a_{g},\left(c_{j}^{\prime}=c_{j}\right.$ for all $\left.j \in U-\{g\}\right)$ and $d \succ c^{\prime}$. By (1), $b^{\prime} \succeq d$ follows from $b_{g}^{\prime}>d_{g}$ and $b_{g}^{\prime}=d_{g}$ for all $j \in C-\{g\}$, and $c^{\prime} \succeq a$ follows from $c_{g}^{\prime} \succeq a_{g}$ and $c_{j}^{\prime}=a_{j}$ for all $j \in C-\{g\}$. Therefore, we obtain: $a \succ b^{\prime}$, $b^{\prime} \succeq d, d \succ c^{\prime}$ and $c^{\prime} \succeq a$, which contradicts the transitivity of $\succeq$.

Suppose (4) holds. Then, by restricted weak continuity and $(d \succ c)$, there exists $d^{\prime} \in X$, such that, $d_{g}^{\prime}<d_{g}=b_{g},\left(d_{j}^{\prime}=d_{j}\right.$ for all $\left.j \in U-\{g\}\right)$ and $d^{\prime} \succ c$. By restricted weak continuity and $\left(d^{\prime} \succ c\right)$, there exists $c^{\prime} \in X$, such that $c_{g}^{\prime}>c_{g}=a_{g}$ and $\left(c_{j}^{\prime}=c_{j}\right.$ for all $\left.j \in U-\{g\}\right)$ and $d^{\prime} \succ c^{\prime}$. By (1), $b \succeq d^{\prime}$ follows from $b_{g}>d_{g}^{\prime}$ and $\left(b_{j}=d_{j}^{\prime}\right.$ for all $\left.j \in C-\{g\}\right)$, and $c^{\prime} \succeq a$ follows 
from $c_{g}^{\prime}>a_{g}$ and $\left(c_{j}^{\prime}=a_{j}\right.$ for all $\left.j \in C-\{g\}\right)$. Consequently, we obtain: $a \succ b, b \succeq d^{\prime}, d^{\prime} \succ c^{\prime}$ and $c^{\prime} \succeq a$, a contradiction of the transitivity of $\succeq$.

The proof for the case where (5) holds is similar to the proof for the case where (3) holds, and we omit it.

Suppose (6) holds. Then, by restricted weak continuity and $(a \succ b)$, there exists $a^{\prime} \in X$, such that $a_{g}^{\prime}<a_{g}=c_{g},\left(a_{j}^{\prime}=a_{j}\right.$ for all $\left.j \in C-\{g\}\right)$ and $a^{\prime} \succ b$; and by restricted weak continuity and $(d \succ c)$, there exists $d^{\prime} \in X$, such that $d_{g}^{\prime}<d_{g}=b_{h},\left(d_{j}^{\prime}=d_{j}\right.$ for all $\left.j \in C-\{g\}\right)$ and $d^{\prime} \succ c$. Noting that $\left(c_{g}>a_{g}^{\prime}\right.$ and $c_{j}=a_{j}^{\prime}$ for all $\left.j \in C-\{g\}\right)$ and $\left(b_{g}>d_{g}^{\prime}\right.$ and $b_{j}=d_{j}^{\prime}$ for all $j \in C-\{g\}$ ), by (1), we obtain $c \succeq a^{\prime}$ and $b \succeq d^{\prime}$. Therefore, we have: $a^{\prime} \succ b, b \succeq d^{\prime}, d^{\prime} \succ c$ and $c \succeq a^{\prime}$, which contradicts the transitivity of $\succeq$.

This completes the proof of Proposition 1.

Proof of Proposition 3. Suppose $\succeq$ satisfies weak dominance, contextdependence (type III), and weak continuity in attributes in $D$. We shall show that this leads to a contradiction.

By weak dominance, there exists an attribute $g \in D$, such that (1) holds. In the rest of this proof, we treat such $g$ as fixed.

Given context dependence (type III), there exist $a, b \in X$, such that (for all $j \in C, a_{j}=b_{j}$ ) and $a \succ b$.

We distinguish three cases: (i) $a_{g}=b_{g}=\beta_{g}$; (ii) $a_{g}=b_{g}=\alpha_{g}$; and (iii) $\beta_{g}<a_{g}=b_{g}<\alpha_{g}$. In case (i), by $a \succ b$ and weak continuity in attributes in $D$, we have $a \succ b^{\prime}$ for some $b^{\prime} \in X$ with $b_{g}^{\prime}>b_{g}=\beta_{g}$ and $b_{j}^{\prime}=b_{j}$ for all $j \in U-\{g\}$. By (1) and noting that $b_{g}^{\prime}>a_{g}=\beta_{g}$ and $b_{j}^{\prime}=a_{j}$ for all $j \in C-\{g\}$, we have $b^{\prime} \succeq a$, an immediate contradiction of $a \succ b^{\prime}$. In case (ii), by $a \succ b$ and weak continuity in attributes in $D$, we have $a^{\prime} \succ b$ for some $a^{\prime} \in X$ with $a_{g}^{\prime}<a_{g}=b_{g}=\alpha_{g}$ and $a_{j}^{\prime}=a_{j}$ for all $j \in U-\{g\}$. By (1), it follows that $b \succeq a^{\prime}$, an immediate contradiction of $a^{\prime} \succ b$. In case (iii), by $a \succ b$ and by a straightforward application of weak continuity in attributes in $D$, we have $a^{\prime \prime} \succ b$ for some $a^{\prime \prime} \in X$ with $a_{g}^{\prime \prime}<a_{g}=b_{g}$ and $a_{j}^{\prime \prime}=a_{j}$ for all $j \in U-\{g\}$. Then, by (1), $b \succeq a^{\prime \prime}$, a direct contradiction of $a^{\prime \prime} \succ b$. 